Proceedings of the Thirteenth Symposium on Theory and Practice of Robots and Manipultors,

(Zakopane, 2000) (Eds.: Morecki, A., Bianchi, G., Rzymkowsky, C.)

Springer, Wien, 2000, pp. 197-203.

\title{
Remote Control of Periodic Robot Motion
}

\author{
Tamás Insperger and Gábor Stépán \\ Department of Applied Mechanics, Budapest University of Technology and Economics, Hungary
}

\begin{abstract}
Remote control of robots often leads to the presence of time delay in the information transmission of the signals in the control loop. Analytical methods are available for the calculation of the maximum critical time delays and control gains when stationary end positions of robots, or constant contact forces between actuators and environment are still stable. When the desired trajectory is periodic, or the desired contact force varies periodically, the non-linearities of the robotic structure take an important role even in the local stability behavior about the desired motion. The non-linear characteristics and the periodic path together lead to parametric excitation, i.e., the stiffness, damping and gain parameters may vary periodically in time. The stability behavior of these systems become intricate in the presence of great time delays. Stability charts are constructed which explain the stability properties of remote periodic force control.
\end{abstract}

\section{Introduction}

The design of robot structures and their control usually neglects the presence of time delays. This approach is often acceptable, in spite of the fact, that time delays always appear in real structures. There are three important sources of these delays: (1) the sampling time of the digital controller; (2) the delay of the signals in the information transmission system; (3) the mechanical structure itself. In case (1), the sampling delay is usually in the range of $10^{-3}-10^{-2}$ seconds and it is combined with a so-called zero order holder. In case (2), the time delay is constant and may vary from $10^{-6}$ to 1 second depending on the distance between the controlled robot and the controller, e.g. in master/slave systems. Consequently, this information delay is often negligible, but it may be crucial, for example, in space applications (see Vertut et al., 1976). In case (3), time delay may arise when the actuator is in elastic contact with the environment along a contact surface (see Stépán, 1997), and the delay is inversely proportional to the relative velocity of the contact surfaces. In this study, case (2) is examined only, but the results can partly be extended for the digital control case (1) due to the physical similarities between the two cases.

There are existing methods to estimate the critical values of the delays in case of robot position/force control when the desired position or contact force is fixed in time (see Stépán and Steven, 1990). The linear variational system of the equations of motion at a trivial solution leads to a system of linear autonomous differential-difference equations. In these equations, the time delay is responsible for the presence of the difference in time, while the differential part comes from the time derivatives in Newton's laws. In spite of the finite degrees of freedom, this system has an infinite dimensional nature, its phase space is infinite dimensional in mathematical sense. Still, the linear system allows us to get conclusion on the local stability of 
the robot control strategy. These investigations are supported by methods developed for autonomous, i.e. time independent systems.

When closed trajectories, periodic motion, or periodic time-varying contact forces are desired, the non-linearities of the robot manipulator structure and those of its elastic and viscous elements result time-varying parameters in the linear system for the small perturbations of the desired motion. If the desired motion in time is 'slow', there is not much difference with respect to the stability limits regarding time delays. In other words, the greater the time delay in the information transmission is, the slower the motion should be for stable operation. This is observed when elderly people having slow reflexes (consequently great time delays) are advised to move slower to avoid fall-over, i.e. to maintain stability (see Cooper and Kojeca, 1994).

The stability properties of those systems where relatively fast periodic motion has to be controlled remotely is still quite unexplored. The analytical study of these systems may partly explain unexpected losses of stability, while it may also lead to new stable control parameter domains since the time-periodic parameters sometimes stabilize otherwise unstable equilibria (see Insperger and Horváth, 2000). However, this physical observation has not been approved yet in case of delayed or retarded dynamical systems.

As a general introductory example, consider the remote control of a robot having the wellknown general nonlinear system of equations of motion in the form

$$
\mathbf{M}(\mathbf{y}) \ddot{\mathbf{y}}+\mathbf{f}(\mathbf{y}, \dot{\mathbf{y}})=\mathbf{Q}(\mathbf{y}, \dot{\mathbf{y}}),
$$

with the general coordinate vector $\mathbf{y}$, the general mass matrix $\mathbf{M}$, coriolis, centrifugal, gravitational, elastic, viscous and other forces all included in $\mathbf{f}$, and with the control force denoted by $\mathbf{Q}$ (see Spong and Vidyasagar, 1989). If the desired motion is periodic with period $T$, that is $\mathbf{y}_{\mathbf{d}}(t)=\mathbf{y}_{\mathbf{d}}(t+T)$, and the control force is constructed from the computed torque $\mathbf{Q}_{\mathbf{d}}(t)$ and the simplest PD compensator, the linear system of equations of motion with respect to the small perturbation $\mathbf{x}=\mathbf{y}-\mathbf{y}_{\mathbf{d}}$ assumes the form

$$
\ddot{\mathbf{x}}(t)+\mathbf{B}(t) \dot{\mathbf{x}}(t)+\mathbf{C}(t) \mathbf{x}(t)=\mathbf{P}(t) \mathbf{x}(t-\tau)+\mathbf{D}(t) \dot{\mathbf{x}}(t-\tau) .
$$

The coefficient matrices may all be periodic with period $T$ originated in the desired motion period. Due to the information delay $\tau$ occurring in the control, the position and velocity errors contain this delay in the compensator. Thus, the above system is a parametrically excited delay-differential equation. In the subsequent sections, preliminary experimental work, a new stability criterion, and stability charts for remote periodic force control are summarized.

\section{Preliminary experiments}

The Newcastle robot was an excellent tool to study hybrid position/force control strategies (see Stépán and Steven, 1990). In case of a one degree of freedom force control implementation, different digital sampling delays were introduced to check the critical proportional gain $P_{c r}$ applied for the force error at the limit of stability. The mechanical model of the structure is presented in Figure 1. The precisely identified parameters of the robot are the mass $m=2500$ [kg], the viscous damping factor $c=32[\mathrm{Ns} / \mathrm{mm}]$, and the linear stiffness of the force sensor $k=$ $44.5[\mathrm{~N} / \mathrm{mm}]$. The experimental stability chart presented in the plane $(\tau, P)$ of the delay and 
Proceedings of the Thirteenth Symposium on Theory and Practice of Robots and Manipultors, (Zakopane, 2000) (Eds.: Morecki, A., Bianchi, G., Rzymkowsky, C.)

Springer, Wien, 2000, pp. 197-203.

the gain parameters in see Stépán and Steven (1990) confirmed the analytical estimation for the critical proportional gain $P_{c r}$ :

$$
0<P<P_{c r}=(2 c) /(3 k \tau) .
$$

For sampling times in the range 7.5-30 [ms], the critical gains are between 19 and 62 . After the loss of stability, the resulting vibration frequencies were in the surprisingly low range of $3.5-6[\mathrm{~Hz}]$ in accordance with the estimated value

$$
f=\sqrt{c /(6 m \tau)} / \pi \text {. }
$$

These results cannot be extended directly for great time delays and time periodic contact forces. This gives the motivation for the following analysis.

\section{Stability analysis of remote force control}

Let us consider the non-linear spring characteristic $k y+k_{3} y^{3}$ in the force control model of the Newcastle robot as shown in Figure 1. The softening spring has a negative coefficient $k_{3}=$ $-6.6\left[\mathrm{~N} / \mathrm{mm}^{3}\right]$.

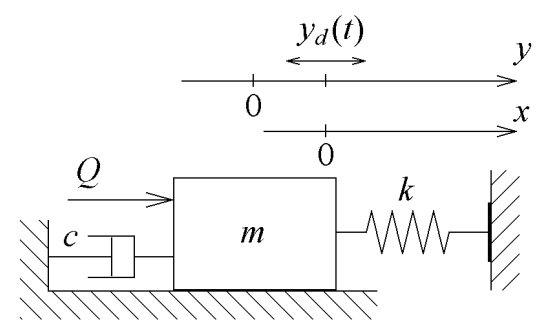

Figure 1. Mechanical model

The equation of motion of force control reads (see Craig, 1986)

$$
m \ddot{y}+c \dot{y}+k y+k_{3} y^{3}=-P F_{e}+\left(Q_{d}+F_{e}\right),
$$

where the force error $F_{e}$ is the difference of the sensed and the desired forces

$$
F_{e}(t)=F_{s}(t)-F_{d}(t)=k y(t-\tau)+k_{3} y^{3}(t-\tau)-\left(k y_{d}(t-\tau)+k_{3} y_{d}{ }^{3}(t-\tau)\right),
$$

and the computed torque assumes the form

$$
Q_{d}(t)=m \ddot{y}_{d}(t)+c \dot{y}_{d}(t)+k y_{d}(t)+k_{3} y_{d}^{3}(t) .
$$

For the small perturbation $x$ defined by $y=y_{d}-x$, the substitutions and linearization leads to the linear time-periodic delay-differential equation

$$
m \ddot{x}(t)+c \dot{x}(t)+\left(k+3 k_{3} y_{d}{ }^{2}(t)\right) x(t)=-(P-1)\left(k+3 k_{3} y_{d}{ }^{2}(t-\tau)\right) x(t-\tau) .
$$

Introduce dimensionless time $t$ by $t=t / \tau$, and by abuse of notation, drop the tilde immediately. We get the following equation 
Proceedings of the Thirteenth Symposium on Theory and Practice of Robots and Manipultors, (Zakopane, 2000) (Eds.: Morecki, A., Bianchi, G., Rzymkowsky, C.)

Springer, Wien, 2000, pp. 197-203.

$$
\begin{array}{r}
\ddot{x}(t)+4 \pi \zeta\left(\tau f_{n}\right) \dot{x}(t)+4 \pi^{2}\left(\tau f_{n}\right)^{2}\left(1+3 r_{k} y_{d}{ }^{2}(t)\right) x(t) \\
=-(P-1) 4 \pi^{2}\left(\tau f_{n}\right)^{2}\left(1+3 r_{k} y_{d}{ }^{2}(t-1)\right) x(t-1),
\end{array}
$$

where

$f_{n}=(\sqrt{k / m}) / 2 \pi \quad$ is the natural frequency of the undamped, uncontrolled oscillating system,

$\zeta=c /(2 \sqrt{\mathrm{km}}) \quad$ is the relative damping factor,

$r_{k}=k_{3} / k \quad$ is a ratio characterising the spring non-linearity.

Clearly, for linear spring characteristic $r_{k}=0$, the system is time independent, and the stability chart can be given in closed form as explained in Section 2 for the preliminary experiments (see Figure 2).

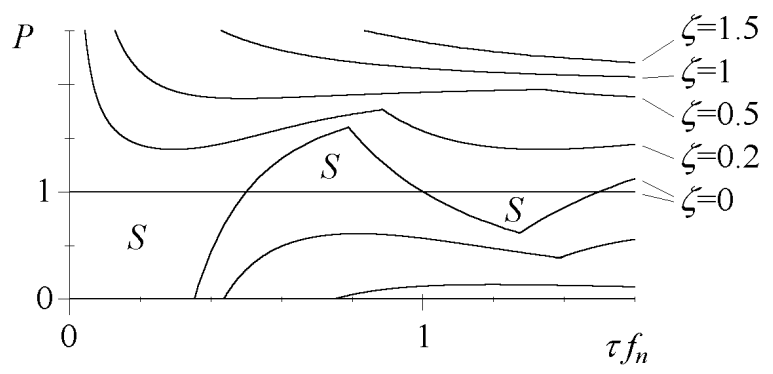

Figure 2. Stability charts for linear spring and various damping values

The stability of fixed point $x(t) \equiv 0$ of equation (2) for a non-linear spring with $r_{k} \neq 0$ cannot be determined in closed form. The delayed term $x(t-1)$ is approximated as follows

$$
x(t-1) \approx \int_{-\infty}^{0} x(t+\vartheta) \cdot w_{n}(\vartheta) d \vartheta,
$$

where $w_{n}(\vartheta)$ is a special weight function series coming from the product of a polynomial and an exponential expression

$$
w_{n}(\vartheta)=(-1)^{n} \frac{n^{n+1}}{n !} \vartheta^{n} \mathrm{e}^{n \vartheta}
$$

The function $w_{n}(\vartheta)$ satisfies the following properties

$$
\int_{-\infty}^{0} w_{n}(\vartheta) d \vartheta=1, \quad \quad \lim _{n \rightarrow \infty} w_{n}(\vartheta)=\delta(\vartheta+1),
$$

where $\delta$ is the Dirac distribution. It was proved by Fargue (1973) that (3) converges to $x(t-1)$ as $n$ tends to infinity. Figure 3 shows the weight function with parameters $n=2,10,50,100$. It can be seen, that the greater the approximation parameter $n$ is, the more correct the approximation is. 
Proceedings of the Thirteenth Symposium on Theory and Practice of Robots and Manipultors, (Zakopane, 2000) (Eds.: Morecki, A., Bianchi, G., Rzymkowsky, C.)

Springer, Wien, 2000, pp. 197-203.

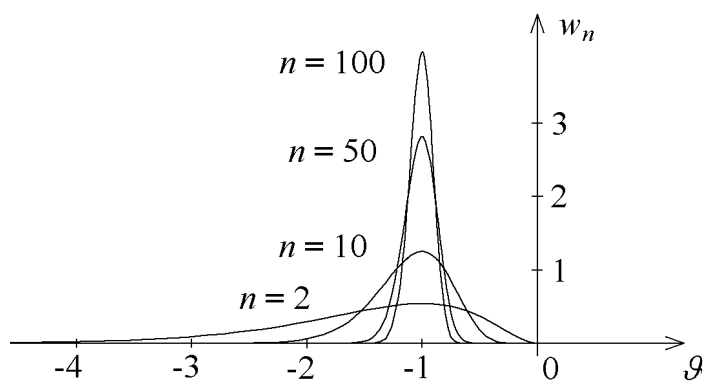

Figure 3. Weight functions

Approximation (3) can be applied in (2). A long calculation (derivations and partial integration) yields a finite dimensional system of differential equations with a time periodic coefficient matrix

$$
\frac{\mathrm{d}}{\mathrm{d} t} \mathbf{z}(t)=\mathbf{A}(t) \mathbf{z}(t)
$$

where $\mathbf{z}(t)=\operatorname{col}\left(z_{1} z_{2} \ldots z_{n+3}\right)$ and $c(t)=4 \pi^{2}\left(1+3 r_{k} y_{d}^{2}(t)\right)$ in the coefficient matrix

$$
\mathbf{A}(t)=\left[\begin{array}{cccccc}
0 & 1 & 0 & 0 & \cdots & 0 \\
-\left(\tau f_{n}\right)^{2} c(t) & -4 \pi \zeta\left(\tau f_{n}\right) & -(P-1)\left(\tau f_{n}\right)^{2} c(t-1) & 0 & \cdots & 0 \\
0 & 0 & -n & -1 & \cdots & 0 \\
\vdots & \vdots & \vdots & \ddots & \ddots & \vdots \\
0 & 0 & 0 & \cdots & -n & -1 \\
(-1)^{n}(n)^{n+1} & 0 & 0 & \cdots & 0 & -n
\end{array}\right] .
$$

System (4) is asymptotically stable if and only if all the characteristic multipliers of the principle matrix $\mathbf{C}$ are in modulus less than one, that is they are inside the unit circle of the complex plane. In general, the principal matrix can not be calculated in closed form. However, if the desired contact force is piecewise constant, the function $c(t)$ is also piecewise constant

$$
F_{d}(t)=\left\{\begin{array}{llll}
F_{1} & \text { if } \quad 0 \leq t<T / 2 \quad & \Rightarrow & c(t) \equiv c_{1} \\
F_{2} & \text { if } \quad T / 2 \leq t<T & \Rightarrow & c(t) \equiv c_{2}
\end{array} .\right.
$$

In this case, the principal matrix takes the form

$$
\mathbf{C}=\exp \left(\mathbf{A}_{\mathbf{4}} t_{4}\right) \exp \left(\mathbf{A}_{\mathbf{3}} t_{3}\right) \exp \left(\mathbf{A}_{2} t_{2}\right) \exp \left(\mathbf{A}_{1} t_{1}\right)
$$

where the functions $c(t)$ and $c(t-1)$ are constant in each time interval of length $t_{i}$ belonging to the coefficient matrix $\mathbf{A}_{\mathbf{i}}\left(\mathrm{i}=1,2,3,4\right.$ and $t_{1}+t_{2}+t_{3}+t_{4}=T$ ). 
Proceedings of the Thirteenth Symposium on Theory and Practice of Robots and Manipultors, (Zakopane, 2000) (Eds.: Morecki, A., Bianchi, G., Rzymkowsky, C.)

Springer, Wien, 2000, pp. 197-203.

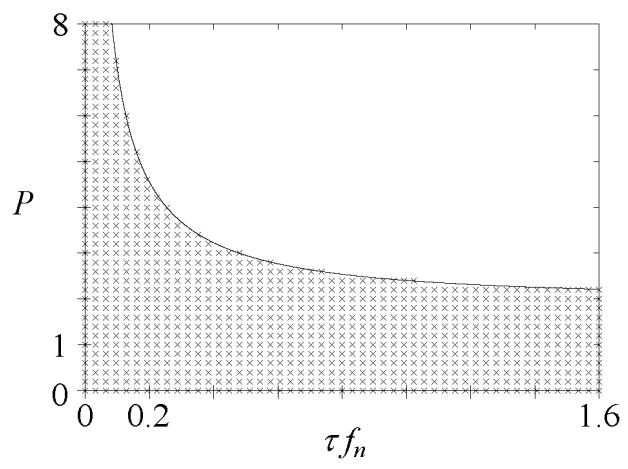

Figure 4. Stability chart for constant desired contact force
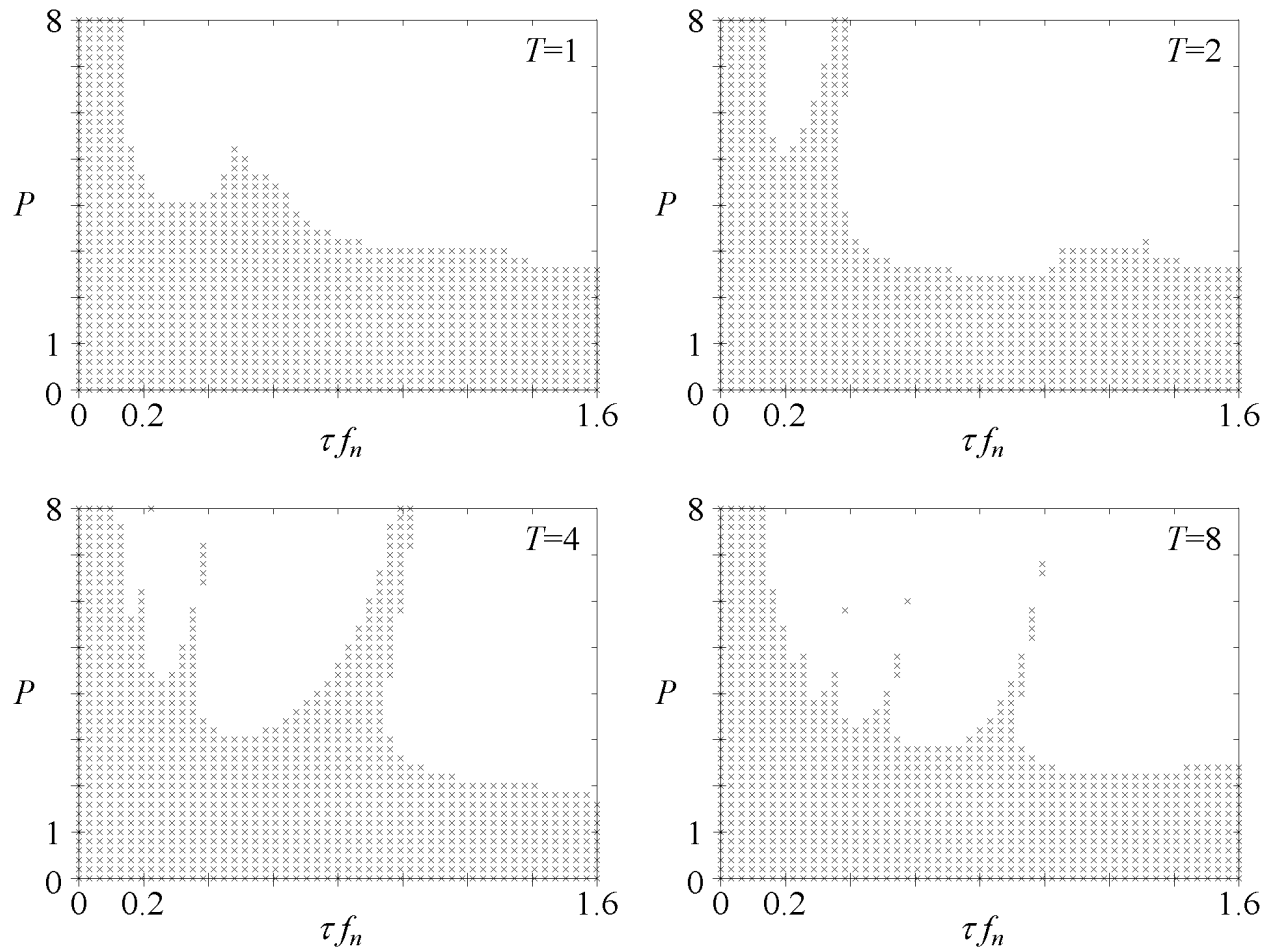

Figure 5. Stability charts for time periodic desired contact forces with various periods $T$ 
Proceedings of the Thirteenth Symposium on Theory and Practice of Robots and Manipultors,

(Zakopane, 2000) (Eds.: Morecki, A., Bianchi, G., Rzymkowsky, C.)

Springer, Wien, 2000, pp. 197-203.

\section{Conclusions}

The system is analysed with the parameters of the Newcastle robot (see in Section 2), the modal parameters are $f_{n}=0.67[\mathrm{~Hz}], \zeta=1.52$. The desired periodic contact force switches between $F_{1}=21.4[\mathrm{~N}], F_{2}=44.5[\mathrm{~N}]$ in $(5)$. The stability charts are shown in the $\left(\mathrm{P},\left(\tau f_{n}\right)\right)$ plane of dimensionless parameters, for various time periods $(T=1,2,4,8[\mathrm{~s}])$. The stable points are marked with crosses in the stability charts of Figures 4 and 5 In case of constant desired force, the $n=100$ approximation gives an acceptable result in the presented parameter domain with errors under $1 \%$ relative to the exactly known stability limit (solid line in Figure 4).

As Figures 5 clearly show, the maximal gains decrease for increasing time delays. For long time periods $T$, the stability properties approach the stationary case shown in Figure 4. For small periods $T$, i.e. for fast changes in the desired contact force, certain ranges of the delay (and natural frequency) show great improvement in the stability properties. These effects, together with the periodic changes in the contact force may greatly help to improve accuracy limited by the Coulomb friction in the structure.

\section{Acknowledgments}

This research was supported by the Hungarian National Science Foundation under grant no. OTKA T030762/99, and the Ministry of Education and Culture grant no. MKM FKFP 0380/97.

\section{References}

Cooper, J. M., Kojeca, D. M. (1994). Relationship of Leg Strength, H-Reflexes and Balance in Young and Elderly Adults - Preliminary Study, In Proceeidngs of Twelve's International Sypmosium on Biomechanics in Sport, Budapest, 157-158.

Craig, J. J. (1986). Introduction to Robot Mechanics and Control, Reading, Addison-Willey.

Fargue, D. (1973). Réducibilité des Systémes héréditaires á des systémes dinamiques, C. R. Acad. Sci. Paris, 277B: 471-473.

Insperger, T., Horváth, R. (2000). Pendulum With Harmonic Variation of the Suspension Point, 2000, Periodica Polytechnica - Mechanical Engineering, 44, Budapest, to appear.

Spong, M. W., Vidyasagar, M. (1989). Robot Dynamics and Controll, Singapure, Wiley \& Sons.

Stépán G. (1997). Nonlinear Oscillations and Shimmying wheels, In Proceedings of Symposium on New Applications of Nonlinear and Chaotic Dynamics in Mechanics, Ithaca, 373-387.

Stépán, G., Steven A. (1990). Theoretical and Experimental Stability Analysis of a Hybrid PositionForce controlled robot, In Proceedings of Eight Symposium on Theory and Practice of Robots and Manipulators, Cracow, 53-60.

Vertut, J., Charles, J., Coiffet, P. and Petit. M. (1976). Advance of the New MA23 Force Reflecting Manipulator System, In Proceedings of the Second Symposium on Theory and Practice of Robots and Manipulators, Udine, 50-57. 